\title{
Transmission Performance of Transparent Multi-Wavelength Optical Cross-connected Networks
}

\author{
G. A. Castañón, O. K. Tonguz and A. Bononi ${ }^{1}$
}

\begin{abstract}
The transmission performance of regular two-connected multi-hop transparent optical networks in uniform traffic under hot-potato, single-buffer deflection routing schemes is presented. Manhattan Street (MS) Network and ShuffleNet (SN) are compared in terms of bit error rate (BER) and packet error rate (PER) both analytically and by simulation. We implement a novel strategy of analysis, in which the transmission performance evaluation is linked to the traffic randomness of the networks. Amplifier spontaneous emission (ASE) noise, and device-induced crosstalk severely limit the characteristics of the network, such as propagation distance, sustainable traffic, and bit-rate. Our results indicate that under the same load the BER performance of single-buffer deflection routing is worse than hot-potato. However, at BER $=10^{-9}$ single-buffer has a higher throughput than hot-potato. It is shown that the feasibility of deflection routing in transparent networks with MS and SN topologies heavily depends on the power coupling coefficient of the routing space switch used in each node.
\end{abstract}

\section{Introduction}

Transparent cross-connected optical networks with deflection routing have recently become the focus of much research [1], [2], [3], [4], [5]. The idea behind transparent networks is to modulate a lightwave carrier with data packets and let these optical packets travel from source to destination through a sequence of intermediate nodes without conversion to electronic form. Cross-connected topologies achieve higher throughput than linear topologies like buses and rings. If buffers are not available, the packets can be temporarily deflected to an undesired link. Thus, deflection routing allows use of fiber links as optical buffers [2]-[4]. However, the accumulation of weak noises such as ASE noise and crosstalk introduced by the $2 \times 2$ space switches and by the wavelength demultiplexers/multiplexers (DEMUX/MUX) in the nodes causes a significant performance degradation in transparent networks.

\footnotetext{
${ }^{1}$ G. A. Castañón, O. K. Tonguz are with the Photonics Research Laboratory, Department of Electrical and Computer Engineering, State University of New York at Buffalo, Buffalo, NY 14260, USA. http://wwwacsu.buffalo.edu/ castanon, http://www.acsu.buffalo.edu/ ${ }^{\sim}$ tonguz.

A. Bononi is with Dipartimento di Ingegneria dell'Informazione Università di Parma, I-43100 Parma ITALY. E-mail: alberto@com.tlc.unipr.it.
}

The traffic performance of multi-hop packet switching networks such as Manhattan Street Network (MS) [1] and ShuffleNet (SN) [2] have been studied extensively [1]-[4]. However, relatively few studies consider the BER performance of these networks [5],[6]. In [5] ultrafast soliton communication is used to evaluate the BER and PER in multi-hop networks with deflection routing, but the impact of crosstalk is not considered. In [6] the BER analysis appearing in [7] and [8] is extended using a semianalytical simulation method for estimating the effect of the interference (intersymbol interference, crosstalk) noises on the BER performance of a circuit switching network. However, the impact of traffic randomness is not considered in the analysis.

This paper presents the first complete BER and PER analysis based on the traffic randomness of multi-hop packet-switched transparent multiwavelength networks. It is shown that the BER performance strongly depends on the traffic load of the network and the transmission power. We present the limit of operation based on a uniform traffic scenario. The main impairments considered in the analysis are intra-band crosstalk and ASE noise.

\section{Node structure}

The node is composed of a stack of submodules, one per wavelength. The wavelengths from the input fibers are spatially demultiplexed and sent to the appropriate submodule for add/drop operations and switching. Packets from the submodules are finally re-multiplexed onto the output fibers. Fig. 1 shows a block diagram of the submodule described in [5]. With hot-potato routing the main switch is a simple cross-bar switch. The scheme for a single-buffer memory is also shown in Fig. 1. A deflection occurs in the single-buffer scheme when three conflicting packets with the same output preference are present, two at the input links of the main switch and one in the buffer. The addition of more optical buffers reduces deflections but introduces more power losses and crosstalk. It will be shown that the presence of a single buffer can significantly degrade the transmission performance.

\subsection{Traffic Parameters}

There are four parameters that determine the transmission performance:

i) Average number of hops $\mathbf{H}$ that packets experience 


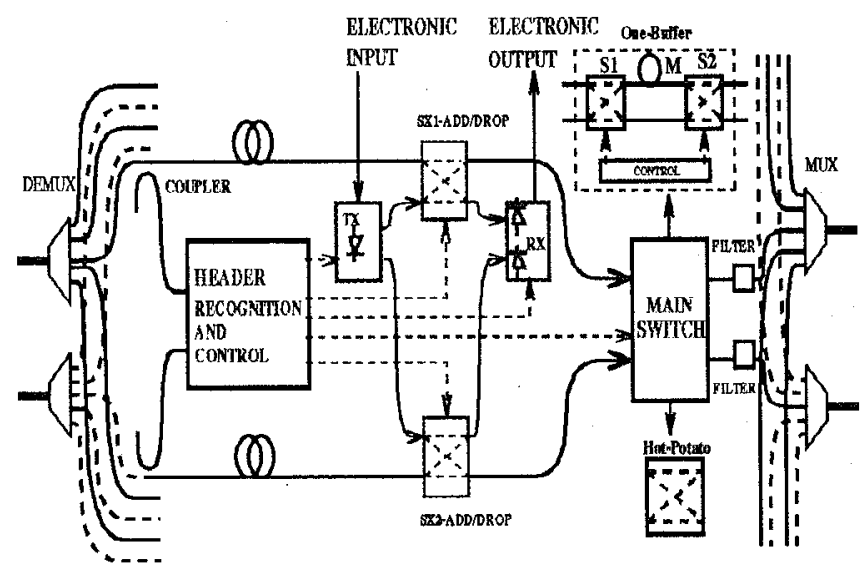

Figure 1: Node block diagram, a) Hot-potato switch, and Onebuffer switch, $M$ is the memory, $S 1$ and $S 2$ are exchange-bypass switches.

before absorption. The probability of packet absorption a is related to $H$ as: $\mathbf{a}=\mathbf{1} / \mathbf{H}[4]$.

ii) The probability mass function (pmf) of the number of hops taken by a typical packet before absorption, shown in Fig. 2a [4].

iii) The pmf of the age of packets, i.e., the number of hops experienced by a typical packet when it visits a generic node. This is depicted in Fig. $2 \mathrm{~b}$ and an analytical method to derive it is presented in [9]. Such curves are important when the amplifier gain is not equal to the inter-amplifier loss, since packets have power variations depending directly on their age. Fig. 3a shows the average age $A$ and Fig. 3b [4] shows $H$ versus the probability of packet generation $\mathrm{g}$.

iv) The link utilization $\mathbf{u}$, i.e., the probability of finding a packet at the input links of a node at each slot. When each submodule has two receivers and one transmitter, the link utilization is [4]

$$
u=\frac{\sqrt{a^{2}+g^{2}(1-a)^{2}}-a}{g(1-a)^{2}}
$$

\section{Device-induced optical crosstalk}

The crosstalk generated in a $2 \times 2$ space switch is due to incomplete switching. A fraction $1-\alpha$ of the signal power exits from the desired port, while a fraction $\alpha$ leaks from the undesired port (see Fig. 1b). If two signals at the same wavelength are present at the inputs, intra-band crosstalk is generated.

A wavelength DEMUX behaves like a prism, that fans out the light from the input fiber into distinct color (wavelength) beams which are coupled to distinct outputs. The crosstalk in the DEMUX is (see Fig. 1) due to residues of light from neighboring colors on each output. This inter-band crosstalk becomes intra-band crosstalk at the multiplexer (MUX), when colors are merged again on the output fiber [6]. The inter-band crosstalk can

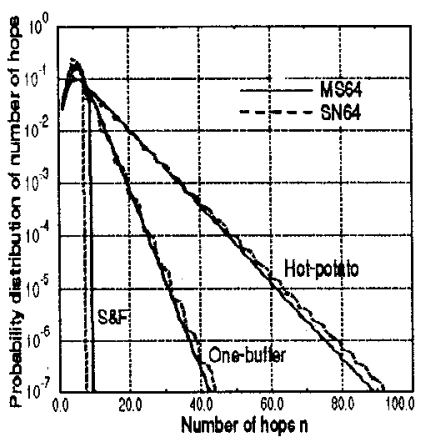

(a)

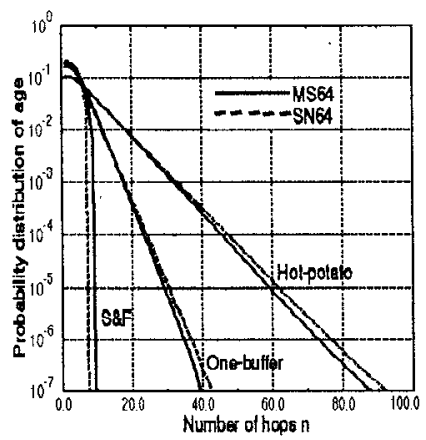

(b)
Figure 2: a)Probability distribution of number of hops versus $n$, b) Probability distribution of the age versus number of hops $n$.

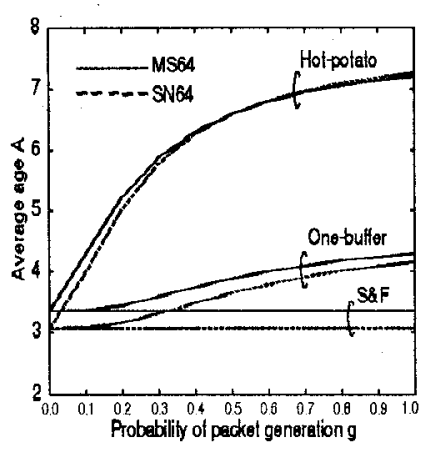

(a)

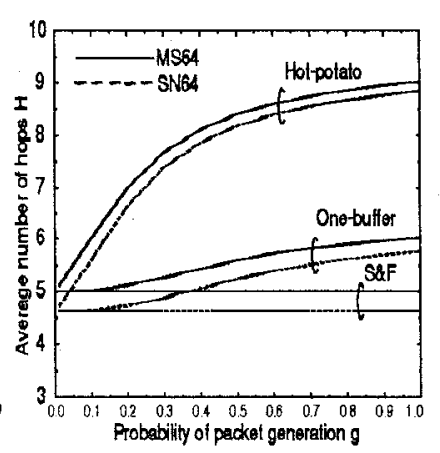

(b)
Figure 3: a) Average age $A$ versus packet generation $g$, b) $H$ versus $g$.

be reduced by placing narrow-band optical filters before multiplexing. The amount of suppression of the interband components will depend on the transfer function $T(\Delta \lambda)$ of the filters.

\section{Signal-to-noise ratio}

The signal-to-noise ratio (SNR) at the output of a directdetection receiver for on-off keying (OOK) modulation format can be expressed as [10]

$$
S N R=\frac{R P_{s i g}}{\sqrt{X(1)}+\sqrt{X(0)}}
$$

where $R=\left(\frac{\eta e}{h \nu}\right)$ is the photodetector responsivity (unit: Ampere/Watt), $\eta$ is the quantum efficiency (unit: dimensionless), $e$ is the electron's charge (unit: Coulomb), and $h \nu$ is the energy of a photon (unit: Joule). $P_{s i g}$ is the desired signal power during a mark, $X(1)=\sigma_{s-x t}^{2}+$ $\sigma_{s-s p}^{2}+\sigma_{x t-x t}^{2}+\sigma_{x t-s p}^{2}+\sigma_{s p-s p}^{2}$, and $X(0)=\sigma_{x t-x t}^{2}+$ $\sigma_{x t-s p}^{2}+\sigma_{s p-s p}^{2}$. The variance terms refer to the signalcrosstalk (s-xt), signal-ASE (s-sp), crosstalk-crosstalk (xtxt), cro- sstalk-ASE (xt-sp), and ASE-ASE (sp-sp) beat terms, respectively. Contribution of shot and thermal noise were not included because they are negligible compared to the other beat terms. 
Assuming that the polarization and phase of the crosstalk signals are uniformly distributed random variables, and mark and space symbols are equally likely, such variances are given by the Apendix:

$$
\begin{gathered}
\sigma_{s-x t}^{2}=R^{2} \frac{P_{s i g} P_{x t}}{2} \\
\sigma_{x t-x t}^{2}=R^{2}(N-1) \frac{P_{x t}^{2}}{8 N} \\
\sigma_{x t-s p}^{2}=2 \frac{B_{e}}{B_{o}} R^{2} P_{x t} P_{a s e}
\end{gathered}
$$

where $B_{e}$ is the electrical filter bandwidth, $B_{o}$ is the optical filter bandwidth, $N$ is the number of crosstalk interferers $(N \geq 1)$, and $P_{x t}$ is the accumulated crosstalk power. $P_{a s e}$ is the accumulated amplifier noise. In the worst case, when one considers polarization matching for all the beat interferers, the terms $\sigma_{x t-x t}^{2}$ and $\sigma_{s-x t}^{2}$ are scaled up by a factor of two. Equations for signal-spontaneous $\left(\sigma_{s-s p}^{2}=\frac{\boldsymbol{R}^{2} 4 B_{c}}{B_{o}} P_{s i g} P_{a s e}\right)$ and spontaneous-spontaneous $\left(\sigma_{s p-s p}^{2}=\frac{R^{2} B_{e}\left(2 B_{o}-B_{e}\right)}{B_{o}^{2}} P_{a s e}^{2}\right)$ beat terms are given in [11] and [10].

\section{Evaluation of crosstalk}

\subsection{Analysis}

This section will derive the necessary equations to compute the signal power, crosstalk, and ASE noise, at any traffic load, of a test packet traveling in a cross-connected network. It is assumed that: i) The bit times of all channels are aligned; ii) All lasers transmit with the same power $P_{T X}$; and iii) The inter-node distance is constant.

An example of the scenario analyzed is shown in Fig. 4. The bold line in Fig. 4 is the path of a test packet sent from node 15 to node 1 , bold-dashed lines are the paths of the interference packets, and narrow lines are generic cross-connections. In this paper only powers that interfere in a direct way with a test packet are considered because power coupling coefficient $\alpha<<1$. The total power $\left(P_{R E, i}\right)$ of the test packet is

$$
\begin{aligned}
P_{R E, i} & =\left(P_{s i g, i}+P_{x t, i}+P_{A S E, i}+P_{x t, i}^{A S E}\right) G_{R E} \\
& +P_{A S E, R E} \quad i=1,2, \ldots
\end{aligned}
$$

where the subscript $i$ represents the number of hops. It can be observed from Fig. 1 that a hop starts and ends after the ADD/DROP switch. $G_{R E}$ is the receiver gain and $P_{A S E, R E}$ is the ASE noise produced by the receiver's amplifier, if a pre-amplified direct-detection receiver is used. The signal power is

$$
P_{s i g, i}=P_{T X} L^{S} L_{\pi}^{i} \quad i=1,2, \ldots
$$

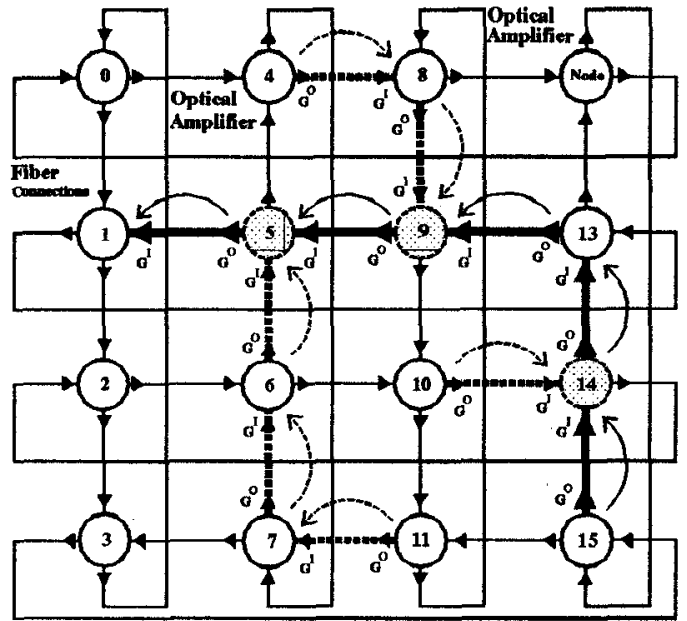

Figure 4: 16-node Manhattan street network. The bold line is the path of a test packet sent from node 15 to node 1, bold-dashed lines are the paths of the interference packets, narrow lines are generic cross-connections. Observe that the test packet experiences crosstalk in three of the nodes (the shadowed nodes 14, 9, and 5) and the interference packets have 1,2, and 3 hops, respectively, as the arc-dashed-arrows indicate. Optical amplifiers are located at the output and at the input of the node. Orientation of the amplifiers also indicate the communication direction. The architecture of each node is the same and is given in Fig. 1

$L_{\pi}=L^{R 1} L^{R 2} L^{M} G^{O} L^{F} G^{I} L^{D} L^{C} L^{S}$ where the righthand side terms are the loss of the first router switch (see Fig. 1), loss of the second router switch (for the case of single-buffer), MUX loss, gain of the output amplifier (see Fig. 4), fiber loss, gain of the input amplifier, DEMUX loss, coupling loss, and ADD/DROP switch loss, respectively. In case of hot-potato $L^{R 2}=1$. If only the output amplifier (input amplifier) is present, then $G^{I}=1\left(G^{O}=1\right)$.

$$
\begin{aligned}
P_{x t, i} & =\left[\left\{\left(P_{x t, i-1}+\Upsilon_{s w 1}\right) L^{R 1}+\Upsilon_{s w 2}\right\} L^{R 2} L^{M}\right. \\
& \left.+\xi_{D-M}\right] G^{O} L^{F} G^{I} L^{D} L^{C} L^{S} \quad i=1,2, . .
\end{aligned}
$$

where $P_{x t, 0}=0$. Eq. (8) represents the accumulation of crosstalk noise. It considers the switch intraband crosstalk and the DEMUX/MUX intraband crosstalk. The first switch crosstalk term is

$\Upsilon_{s w 1}= \begin{cases}u(1-a) P_{x t, A} & \text { if } i=1 \\ u(1-a) P_{x t, A} & \\ +[u a g+(1-u) g] P_{X T} L^{S} \alpha & \text { otherwise. }\end{cases}$

When $i=1$ the crosstalk statistic represents the probability of having a packet at the input of the main switch block (see Fig. 1) given that a test packet was generated by a node. This event occurs if a packet is present and not absorbed. When $i>1$ we add the crosstalk probability of a new generated packet present at the input of the main switch. Hence, $u a g+(1-u) g$ 
represents the probability of having a packet present, the packet absorbed, and a new packet generated, or the input link is empty and a new packet is generated. In (9) the crosstalk produced by the ADD/DROP switch has been neglected because the probability of adding and dropping a packet at the same time and by the same switch is negligible.

Now, when use is made of a single-buffer optical memory proposed in [4], the second switch crosstalk term is

$$
\Upsilon_{s w 2}=u P_{x t, A} L^{R 1}
$$

where the probability of memory being full can be approximated as $p_{m} \simeq u$. Also, the probability of packet crosstalk at the second switch can be approximated as $p_{m}$

The DEMUX-MUX intra-band crosstalk is

$$
\xi_{D-M}= \begin{cases}\frac{u^{2}(1-a)}{2} N\left(P_{s i g, A}+P_{x t, A}\right) & \\ \Psi_{D} T(\Delta \lambda) L^{R 1} L^{R 2} L^{M} & \text { if } i=1 \\ (1+u) \frac{u(1-a)}{2} N\left(P_{s i g, A}+P_{x t, A}\right) & \\ \Psi_{D} T(\Delta \lambda)^{R 1} L^{R 2} L^{M} & \text { if } i>1\end{cases}
$$

where $u(1-a) / 2$ is the probability of having an adjacent packet at the adjacent channel, such that it is not absorbed, and it is routed with the test packet with probability 0.5 . Also $u^{2}(1-a) / 2$ is the probability of having a packet of the same wavelength, which is equal to $u$, at the second fiber multiplied by $u(1-a) / 2$. In eq. (11) it is assumed that in the "empty" slots the ADD/DROP switch is always in drop position to drain the noises from the network. In (11) the adjacent channel inter-band crosstalk produced by the demultiplexer is represented by $\Psi_{D}$. The factor $\aleph$ can take values of 2 or 1 depending on the number of physical adjacent channels.

The ASE $\left(P_{A S E, i}\right)$ noise is the one produced by the chain of optical amplifiers, and ASE crosstalk noise of the interference packets $\left(P_{x t, i}^{A S E}\right)$. Hence,

$$
\begin{aligned}
P_{A S E, \dot{\mathbf{b}}} & =\left(P_{A S E, i-1} L_{\beta}+P_{s p}^{O} L^{F} G^{I}\right. \\
& \left.+P_{s p}^{I}\right) L^{D} L^{C} L^{S} \quad i=1,2, \ldots
\end{aligned}
$$

where $L_{\beta}=L^{R 1} L^{R 2} L^{M} G^{O} L^{F} G^{I}, P_{A S E, 0}=0, P_{s p}^{I / O}=$ $n_{s p} m h v\left(G^{I / O}-1\right) B$ [12], $m$ is the effective number of modes, $n_{s p}$ is the spontaneous emission factor, $h v$ is the energy of a photon, and $B$ is the optical bandwidth. If only the output amplifier is present $P_{s p}^{I}=0$ and vice versa. The accumulation of ASE crosstalk noise can be derived following the logic of equations (8) to (11). The ASE crosstalk statistics are very similar to the signal crosstalk statistics. However, eq. (12) is a good approximation to the total ASE noise power.

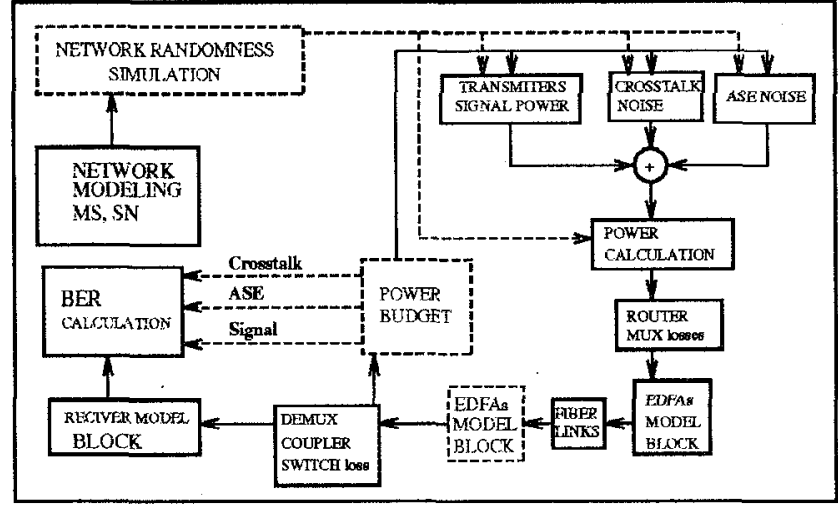

Figure 5: Block diagram of the simulation strategy.

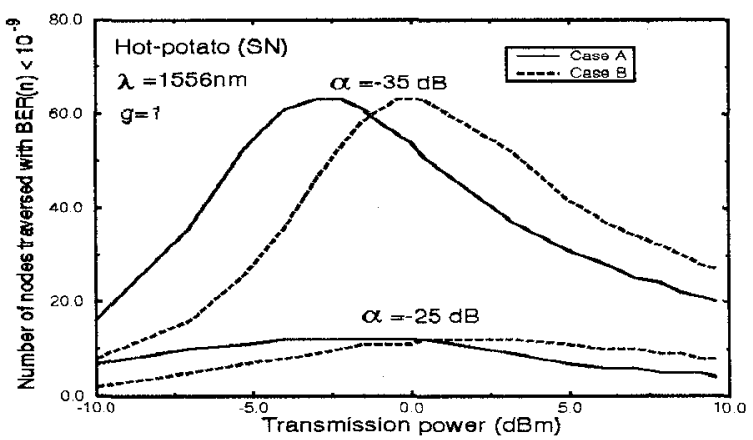

Figure 6: Maximum number of nodes traversed by a signal with a $\mathrm{BER}<10^{-9}$ versus input power at $\mathrm{g}=1$.

Eq. (8) is a recursive transcendental equation for $P_{x t, A}$, and can be solved numerically using a first order approximation $P_{x t, A} \cong \frac{\alpha P_{s i g, A}}{(1-\alpha)}$. However, this value of $P_{x t, A}$ is a good approximation in itself, whereby the contribution of DEMUX/MUX crosstalk is neglected.

\subsection{Simulation Analysis}

The block diagram of the simulation is shown in Fig. 5 . Crosstalk is modeled according to the traffic parameters of the network; i.e., g, a, u probabilities, pmf of the age of the packets, architecture of the submodules and routing algorithm [13]. The signal, crosstalk and ASE noise powers are computed by the power calculation block. The power levels are affected by the respective network losses. A well-known EDFA model [12] is used to compute the output powers according to the characteristics of a real optical amplifier. The BER is processed by the routine for the BER calculation depending on the signal, crosstalk, and ASE noise powers.

\section{Results}

In deflection routing the BER is obtained by conditioning the $\operatorname{BER}(\mathbf{n})$ on the number of hops $\mathbf{n}$ taken by a 


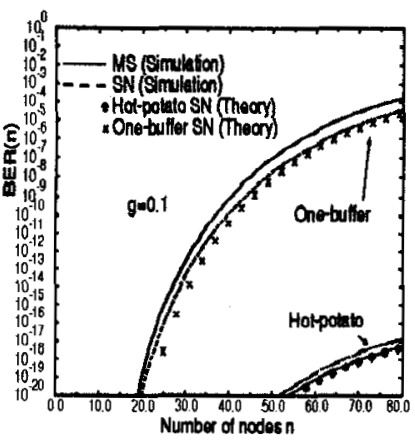

(a)

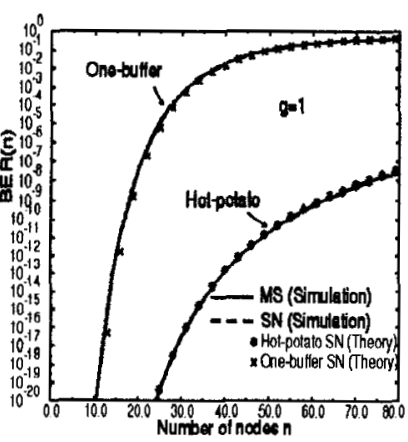

(b)

Figure 7: a)BER(n) versus number of nodes for $g=0.1$. Curves are for wavelength $1556 \mathrm{~nm}$, b) same as a) with $\mathrm{g}=1$.

typical packet as [5]

$$
B E R=\sum_{n=1}^{\infty} B E R(n) P(n) .
$$

The hop distribution $\mathrm{P}(\mathrm{n})$ (Fig. 2) depends on network topology, routing, and load, while the conditional BER(n) depends on the traffic load and the optical characteristics of the network. Similarly $P E R=\sum_{n=1}^{\infty}\{1-$ $\left.[1-B E R(n)]^{M}\right\} P(n)$ [5] where $M$ is the number of bits in the packet.

We analyzed a network with four $2.5 \mathrm{~Gb} / \mathrm{s}$ channels in the range of $1550 \mathrm{~nm}$ to $1556 \mathrm{~nm}$, with $2 \mathrm{~nm}$ channel separation. DEMUX with adjacent signal inter-band crosstalk of $-30 \mathrm{~dB}$, and $2 \times 2$ crossbar optical switch with $\alpha$ between $-25 \mathrm{~dB}$ and $-35 \mathrm{~dB}$ are assumed. Filters have a transfer function $T(\Delta \lambda)=-17 \mathrm{~dB}$. We represent each amplifier by using the spectrally resolved numerical model of [12] with a forward pumping scheme. The absorption and gain parameters are the same as those of [14](see fiber 2a in [14]) with a length of $20 \mathrm{~m}$ and a pump power of $50 \mathrm{~mW}$. Thus, it is assumed that the EDFA's are operating in the saturated regime. A bandwidth of $125 \mathrm{GHz}$ is used to resolve the effect of ASE spectrum. The optical filter at the receiver has a $0.2 \mathrm{~nm}$ bandwidth and the electric filter has a $2.5 \mathrm{GHz}$ bandwidth. We assumed a fiber with dispersion coefficient $D_{c}=1 \mathrm{ps} / \mathrm{km}-\mathrm{nm}$, a loss coefficient of $0.2 \mathrm{~dB} / \mathrm{km}$, an inter-node distance of $15 \mathrm{~km}$, a total node loss of $12.5 \mathrm{~dB}$ for hot-potato and $15.5 \mathrm{~dB}$ for single-buffer node architectures. The loss per single $2 \times 2$ switch is $3 \mathrm{~dB}$. Two cases of the optical amplifier locations are analyzed: output amplifier location (A) and input amplifier location (B).

We optimized each configuration based on the input power. Thus, Fig. 6 shows the maximum number of nodes traversed by a packet with a conditional BER(n) $<10^{-9}$ versus input power at $\mathbf{g}=\mathbf{1}$ for the channel at $1556 \mathrm{~nm}$, one with the worst gain. Results are shown for Hot-potato under SN topology with $\alpha=-35 \mathrm{~dB}$ and -25

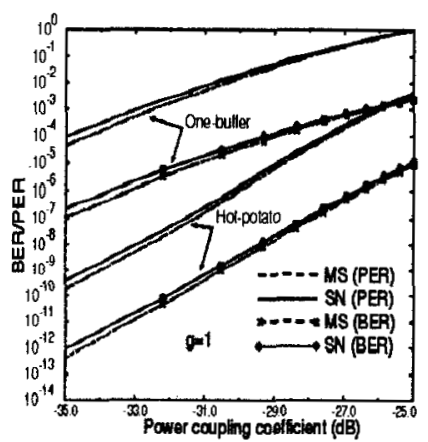

(a)

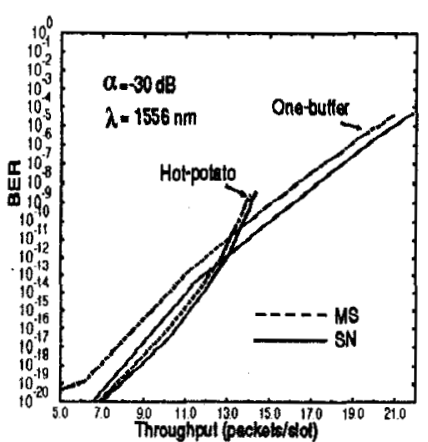

(b)
Figure 8: a) Bit error probability BER and packet error rate (PER) versus power coupling coefficient $\alpha$ for $g=1$. b) BER versus throughput for networks with 64 nodes at $\alpha=-30 \mathrm{~dB}$.

dB. Observe that the BER(n) performance is affected by the value of the power coupling coefficient $\alpha$. For low input power the predominant beat noise is signal-ASE that increase with bit rate and for high input powers the signal-crosstalk limits the network, a noise that is bitrate-independent. The reason that (A) performs better than (B) for low input power is that (A) keeps the signal power at a good level because it is amplified after the node. In the case of (B) the signal power is attenuated by the fiber span loss and then amplified, getting a lower power level with respect to case (A) and in this way affecting the $B E R(n)$. Since configuration (A) require less input power than (B), we will only consider case (A) in the remainder of this paper.

Fig. 7a shows theoretical and simulation results for the conditional BER(n) versus number of nodes traversed by a packet when $g=0.1$ for all the submodules of a network with 64 nodes. We used the Q(SNR) function approximation [15] to obtain the BER(n). Observe that SN and MS have a very similar BER(n) performance. The reason is that the link utilization probability $\mathbf{u}$ and the average age $\mathbf{A}$ of the packets of both topologies are similar.

Fig. $7 \mathrm{~b}$ shows results for the case when $\mathrm{g}=1$. Observe that the $\operatorname{BER}(\mathrm{n})$ degrades with full load. This is because there are more packets in the network and the crosstalk probability increases. The two main reasons why single-buffer has a worse BER(n) than hot-potato are: single-buffer has two optical switches to route the packets, therefore more intraband crosstalk; the second is that ASE noise increases because the EDFA's gain increases (recall that the optical amplifiers are operating in the saturated mode) trying to compensate the loss introduced by the the addition of optical switches. Thus, greater number of optical switches increase the amount of crosstalk and loss. Therefore, using more optical buffers to reduce deflection will deteriorate the 
BER(n) substantially.

Fig. 8a shows BER (using eq. (13)) and PER results versus $\alpha$ for a network of 64 nodes at $\mathrm{g}=1$. Observe that BER of single-buffer is always higher than $10^{-9}$ at full load. Otherwise, hot-potato depends on the power coupling coefficient. For $\alpha<-30.5 \mathrm{~dB}$ the BER $<10^{-9}$ and vice versa. However, for a fair comparison of hot-potato and single-buffer, Fig. $8 \mathrm{~b}$ shows BER versus throughput [4]. Observe that at $\mathrm{BER}=10^{-9}$ single-buffer has a higher throughput than hot-potato due to the superior teletraffic performance of single-buffer. Also, observe that under the same throughput $\mathrm{SN}$ has a better BER performance than MS due to the fact that SN has less traffic congestion because the link load is lower and the packet absorption probability is higher.

\section{Conclusions}

Crosstalk and ASE noise limit the propagation distance, traffic load and bit rate performance of the network. Our results indicate that under the same throughput SN has a better BER performance than MS. Results show that hot-potato deflection routing has a better BER performance than single-buffer at low throughput. However, at $\mathrm{BER}=10^{-9}$ single-buffer has a higher throughput than hot-potato due to the fact that single-buffer has less traffic congestion. If the bit rate increases the BER performance is worse than the shown, but if it decreases the BER improves making possible the operation of singlebuffer at high throughput. However, if no special precautions (such as optimizing the transmission power) are taken to reduce the intra-band crosstalk, SN and MS networks will be limited to a few hops.

The feasibility of deflection routing in transparent networks will heavily depend on the isolation characteristics of the optical switching elements. The most crucial component is the routing space switch. A high power coupling coefficient $\alpha$ limits the operation of the network, while a low $\alpha$ will make its operation possible with good performance.

\section{APPENDIX}

In this section we will derive the signal-crosstalk beat noise $\sigma_{s-x t}^{2}$, crosstalk-crosstalk beat noise $\sigma_{x t-x t}^{2}$, and crosstalk-spontaneous beat noise $a_{x t-s p}^{2}$. We will consider that the phase differences have a uniform random distribution. Also, we will assume a uniform random polarization for each linearly polarized component of light that impinges on the photodetector.

The total electric field at the input of the photodetector is

$$
\begin{aligned}
E(t) & =R c\left[\vec{p}_{s} \sqrt{2 P_{s i g} j}\left(w_{s} t+\phi_{s}\right)\right. \\
& \left.+\sum_{i=1}^{N} \vec{p}_{x t, i} \sqrt{2 P_{x t, i}} e^{j\left(w_{i} t+\phi_{i}\right)}\right]+N(t)
\end{aligned}
$$

where $P_{s i g}$ is the signal power, $\vec{p}_{s}$ is the polarization direction vector of the signal, $w_{s}=2 \pi f_{s}$ is the optical signal carrier frequency, and $\phi_{s}$ is the random phase. $P_{x t, i}$ is the ith crosstalk power, $\vec{p}_{x t, i}$ is the polarization direction vector of the ith crosstalk component, $w_{i}=2 \pi f_{i}$ is the respective optical frequency and $\phi_{i}$ is the random phase uniformly distributed in $[-\pi, \pi] . N(t)$ is the amplifier spontaneous emission noise, assumed to be zero mean, gaussian, with autocorrelation $R_{N}(\tau)=E[N(t) N(t+\tau)]$ and two sided spectral density [11]

$$
S_{N}(f)=\int_{-\infty}^{\infty} R_{N}(\tau) e^{-j w \tau} d \tau
$$

The total power incident on the detector includes direct detection terms that may be written by

$$
P_{s i g,(x t, i)}(t)=\frac{1}{2}\left[E_{s i g,(x t, i)}(t) \bullet E_{s i g,(x t, i)}^{*}(t)\right]
$$

and signal cross-terms written by [16]

$$
P_{x}(t)=\operatorname{Re}\left[E_{s i g,(x t, i)}(t) \bullet E_{s i g,(x t, i)}^{*}(t)\right]
$$

hence

$$
\begin{aligned}
& P(t)=\left[P_{s i g}+\sum_{i=1}^{N} P_{x t, i}+N(t)^{2}\right. \\
& +2 \sqrt{2 P_{s i g}} N(t) \cos \left(w_{s} t+\phi_{s}\right) \\
& +\sqrt{2 P_{s i g}} \sum_{i=1}^{N} \sqrt{2 P_{x t, i}} \cos \left(\left(w_{s}-w_{i}\right) t+\phi_{s}-\phi_{i}\right) \cdot \cos \Theta_{s, i} \\
& +\sum_{i=1}^{N-1} \sum_{j=i+1}^{N} \sqrt{2 P_{x t, i}} \sqrt{2 P_{x t, j}} \cos \left(\left(w_{i}-w_{j}\right)^{t}+\phi_{i}-\phi_{j}\right) \cdot \cos \Theta_{i, j} \\
& \left.+2 \sum_{i=1}^{N} \sqrt{2 P_{x t, i}} N(t) \cos \left(w_{i} t+\phi_{i}\right)\right]
\end{aligned}
$$

where $\Theta_{i, j}$ is the difference of the polarization angles of the two fields. The mean power is

$$
E[P(t)]=\left[P_{s i g}+\sum_{i=1}^{N} P_{x t, i}+R_{N}(0)\right]
$$

The autocovariance of $P(t)$ defined as

$$
L_{p}(\tau)=E[P(t) P(t+\tau)]-\{E[P(t)]\}^{2}
$$

can be computed to be

$$
\begin{aligned}
& L_{p}(\tau)=4 P_{s i g} R_{N}(\tau) \cos \left(w_{s} \tau\right)+2 R_{N}^{2}(\tau) \\
& +2 P_{s i g} \sum_{i=1}^{N} P_{x t, i} \cos \left(\left(w_{s}-w_{i}\right) \tau\right)<\cos ^{2} \Theta_{s, i}> \\
& +2 \sum_{i=1}^{N-1} \sum_{j=i+1}^{N} P_{x t, i} P_{x t, j} \cos \left(\left(w_{i}-w_{j}\right) \tau\right)<\cos ^{2} \Theta_{i, j}> \\
& +4 \sum_{i=1}^{N} P_{x t, i} R_{N}(\tau) \cos \left(w_{i} \tau\right)
\end{aligned}
$$

where the relation [17] $E\left[N^{2}(t) N^{2}(t+\tau)\right]=R_{N}^{2}(0)+2 R_{N}^{2}(\tau)$ was used since $N(t)$ is Gaussian. For uniformly distributed polarization angles we have $\left\langle\cos ^{2} \Theta_{i, j}\right\rangle=1 / 2$. When the polarizations match, the beat interference effects are twice as severe.

The autocovariance of the current $i(t)$ is given by [11]

$$
L_{i}(\tau)=\frac{\eta e^{2}}{h \nu} E[P(t)] \delta(\tau)+\left(\frac{n e}{h \nu}\right)^{2} L_{p}(\tau)
$$

and the spectral density of the photo-current, which is the Fourier transform of $L_{i}(\tau)$, is 


$$
\begin{aligned}
& S_{i}(f)=\frac{\eta e^{2}}{h \nu}\left[P_{s i g}+\sum_{i=1}^{N} P_{x t, i}+R_{N}(0)\right] \\
& +\left(\frac{\eta e}{h \nu}\right)^{2} 2 P_{s i g}\left[S_{N}\left(f-f_{s}\right)+S_{N}\left(f+f_{s}\right)\right] \\
& +\left(\frac{\eta e}{h \nu}\right)^{2}\left[2 S_{N}(f) \otimes S_{N}(f)\right] \\
& +\left(\frac{\eta e}{h \nu}\right)^{2} \sum_{i=1}^{N} \frac{P_{s i g} P_{x t, i}}{2}\left[\delta\left(f+f_{s}-f_{i}\right)+\delta\left(f+f_{i}-f_{s}\right)\right] \\
& +\left(\frac{\eta e}{h \nu}\right)^{2} \sum_{i=1}^{N-1} \sum_{j=i+1}^{N} \frac{P_{x t, i} P_{x t, j}}{2}\left[\delta\left(f+f_{i}-f_{j}\right)+\delta\left(f+f_{j}-f_{i}\right)\right] \\
& +\left(\frac{\eta e}{h \nu}\right)^{2} \sum_{i=1}^{N} 2 P_{x t, i}\left[S_{N}\left(f-f_{i}\right)+S_{N}\left(f+f_{i}\right)\right]
\end{aligned}
$$

where the first term represents the shot-noise, the second term is the signal-spontaneous beat noise, the third term is spontaneousspontaneous beat noise[10] the fourth term is the signal-crosstalk, the fifth term is the crosstalk-crosstalk, and the last term is the crosstalk-spontaneous. Let us assume that the amplifier noise spectrum is centered at $f_{s}$, and is white over an optical bandwidth of $B_{o}<2 f_{s}$, that is

$$
S_{N}(f)= \begin{cases}\frac{P_{a s e}}{2 B_{0}} & \left|f \pm f_{s}\right| \leq \frac{B_{0}}{2} \\ 0 & \text { otherwise }\end{cases}
$$

where $P_{\text {ase }}$ is the accumulation of ASE noise in a chain of optical amplifiers in $n$ hops within a bandwidth $B_{0}$.

The photo-current $i(t)$ is passed through a low-pass filter with bandwidth $B_{e}$. In order to obtain the powers from (A7), we integrate the power spectral densities between $-B_{e}$ and $B_{e}$, resulting in

$$
\begin{aligned}
\sigma_{s h o t}^{2} & =\frac{\eta e^{2}}{h \nu}\left[P_{s i g}+\sum_{i=1}^{N} \frac{P_{x t, i}}{2}+P_{a s e}\right] 2 B_{e} \\
& =\frac{\eta e^{2}}{h \nu}\left[P_{s i g}+\frac{P_{x t}}{2}+P_{a s e}\right] 2 B_{e}
\end{aligned}
$$

where the $1 / 2$ value is present because $P_{x t, i}$ can be " 0 " or " 1 " with the same probability, and $P_{x t}=\sum_{i=1}^{N} P_{x t, i}$. The signal-crosstalk $\left(\sigma_{s-x t}^{2}\right)$ beat noise power is

$$
\sigma_{s-x t}^{2}=\left(\frac{\eta e}{h \nu}\right)^{2} \sum_{i=1}^{N} \frac{P_{s i g} P_{x t, i}}{2}=\left(\frac{\eta e}{h \nu}\right)^{2} \frac{P_{s i g} P_{x t}}{2}
$$

The crosstalk-crosstalk $\left(\sigma_{x t-x t}^{2}\right)$ beat noise power is

$$
\begin{aligned}
\sigma_{x t-x t}^{2} & =\left(\frac{\eta e}{h \nu}\right)^{2} \sum_{i=1}^{N-1} \sum_{j=i+1}^{N} \frac{P_{x t, i} P_{x t, j}}{4} \\
& =\left(\frac{\eta e}{h \nu}\right)^{2}(N-1) \frac{P_{x t}^{2}}{8 N}
\end{aligned}
$$

where we assumed that $P_{x t, i}=P_{x t, j}=\frac{P_{x i}}{N}$. The crosstalkspontaneous $\left(\sigma_{x t-s p}^{2}\right)$ beat noise power is

$$
\begin{aligned}
\sigma_{x t-s p}^{2} & =2\left(\frac{\eta e}{h \nu}\right)^{2} \sum_{i=1}^{N}\left[\frac{P_{x t, i}}{2} \frac{P_{a s e}}{B_{o}}\right] 2 B_{e} \\
& =2\left(\frac{\eta e}{h \nu}\right)^{2} \frac{B_{e}}{B_{o}} P_{x t} P_{a s e}
\end{aligned}
$$

Expressions for signal-spontaneous $\left(\sigma_{s-s p}^{2}\right)$ and spontaneousspontaneous $\left(\sigma_{s p-s p}^{2}\right)$ are given in $[10]$ and [11].

\section{References}

[1] R. Krishnan and N. F. Maxemchuk, "Is there life beyond linear topologies? a comparison of DQDB and the manhattan street network," in Proc. IEEE INFOCOM'93, pp. 690-698, 1993.

[2] A. S. Acampora, M. J. Karol, and M. G. Hluchyj, "Terabit lightwave networks: the multihop approach," AT\& T Technical Journal, vol. 66, no. 6, pp. 21-34, December 1987.

[3] I. Chlamtac and A. Fumagalli, "An optical switch architecture for manhattan networks," IEEE J. Select Areas in Communication, vol. 11, no. 4, pp. 550-559, May 1993.

[4] F. Forghieri, A. Bononi, and P. Prucnal, "Analysis and comparison of hot-potato and single-buffer deflection routing in very high bit rate optical mesh networks," IEEE Trans. on Commun., vol. 43, no. 1, pp. 88-98, January 1995.

[5] A. Bononi, F. Forghieri, and P. Prucnal, "Design and channel constrain analysis of ultrafast multihop all-optical networks with deflection routing employing solitons," IEEE J. Lightwave Technol., vol. 11, no. 12, pp. 2166-2176, Dec. 1993.

[6] J. Zhou, R. Cadeddu, E. Casaccia, C. Cavazzoni, and M. J. O'Mahony, "Crosstalk in multi-wavelength optical crossconnect networks," IEEE J. Lightwave Technol., vol. 14, no. 6 , pp. $1423-1435,1996$.

[7] J. Zhou, M. J. O'Mahony, and S. D. Walker, "Analysis of optical crosstalk effects in multi-wavelength switched networks," IEEE Photon. Technol. Lett., vol. 6, no. 2, pp. 302-305, 1994.

[8] E. L. Goldstein, L. Eskildsen, and A. Elrefaie, "Performance implications of component crosstalk in transparent lightwave networks," IEEE Photon. Technol. Lett., vol. 6, no. 5, pp. $657-660,1994$.

[9] G. A. Castanon, O. K. Tonguz, and A. Bononi, "BER performance of multi-wavelength optical cross-connected networks with deflection routing," IEE Proc.-Communications, accepted for publication, 1996.

[10] N. A. Olsson, "Lightwave systems with optical amplifiers," IEEE J. Lightwave Technol., vol. 7, no. 7, pp. 1071-1082, July 1989.

[11] R. Ramaswami and P. A. Humblet, "Amplifier induced crosstalk in multichannel optical networks," IEEE J. Lightwave Technol, vol. 8, no. 12, pp. 1882-1896, December 1990.

[12] C. R. Giles and E. Desuvire, "Modeling erbium-doped fiber amplifiers," IEEE J. Lightwave Technol., vol. 9, no. 2, pp. 271-283, Feb. 1991.

[13] N. F. Maxemchuk, "Comparison of deflection and storeand-forward techniques in the manhattan street and shuffeexchange networks," in. Proc. IEEE INFOCOM '89, pp. 800 809, Apr. 1989.

[14] C. R. Giles, C. Burrus, D. DiGiovanni, N. Dutta, and G. Raybon, "Characterization of erbium-doped fiber and application to modeling 980-nm and 1480-nm pumped amplifiers," IEEE Photon. Technol. Lett., vol. 3, pp. 363-365, 1991.

[15] O. K. Tonguz and L. G. Kazovsky, "Theory of directdetection lightwave receivers using optical amplifiers," IEEE J. Lightwave Technol, vol. 9, no. 2, pp. 174-181, Feb. 1991.

[16] M. Born and E. Wolf, Principles of Optics. Pergamon Press, fifth ed., 1975. chap. 7.

[17] A. Papoulis, Probability, Random Variables, and Stochastic Proceses. New York:McGraw-Hill, 2nd ed., 1984. chap. 9. 